\title{
Lectin Histochemical Detection of N-Acetyl Glucosamine and L-Fucose Containing glycoconjugates in Lung Carcinoma
}

\author{
Abbas Ali Niazi ${ }^{1}$, Mohammad Hossein Heidari ${ }^{2,{ }^{*}}$, Yousef Arab $^{3}$, Maryam Arab ${ }^{3}$, Mohammad Reza \\ Arab $^{4}$, Narjes Sargolzaei ${ }^{5}$, Sima Tavakolinezhad ${ }^{6}$ and Fereydoon Sargolzaeiaval ${ }^{6}$ \\ ${ }^{1}$ Department of Pathology, School of Medicine, Zahedan University of Medical Sciences, Zahedan, Iran \\ ${ }^{2}$ Proteomics Research Center, Shahid Beheshti University of Medical Sciences, Tehran, Iran \\ ${ }^{3}$ Ali Ebne Abitaleb Hospital, Zahedan University of Medical Sciences, Zahedan, Iran \\ ${ }^{4}$ Cellular and Molecular Research Center, Resistant Tuberculosis Institute, Department of Anatomical Sciences, School of Medicine, Zahedan University of Medical sciences, \\ Zahedan, Iran \\ ${ }^{5}$ Department of Community Medicine, School of Medicine, Zahedan University of Medical Sciences, Zahedan, Iran \\ ${ }^{6}$ Department of Anatomy, School of Medicine, Zahedan University of Medical Sciences, Zahedan, Iran \\ "Corresponding author: Department of Basic Sciences, School of Allied Medical Sciences and Proteomics Research Center, Shahid Beheshti University of Medical Sciences, \\ Tehran, Iran. Email: mh.heidari.sbmu@gmail.com
}

Received 2020 September 26; Revised 2020 October 12; Accepted 2020 October 19.

\begin{abstract}
Background: Lung cancer has the highest frequency among cancers worldwide. It is the leading cause of cancer-induced death in industrialized countries. Abnormal glycosylation of cell surface and extracellular matrix glycoconjugates are among the most critical issues in neoplasia.

Objectives: This present study aimed to detect $\mathrm{N}$ - acetyl glucosamine (GlcNac) and L-fucose (L-fuc) containing glycoconjugates in lung cancer.

Methods: In this cross-sectional study, we selected paraffin blocks belonging to 25 patients with lung cancer from their pathology files at the Ali-Ebne Abitaleb Hospital, Zahedan, Iran. Six $\mu \mathrm{m}$ sections were obtained from the blocks and stained with HematoxylinEosin (H-E) and lectin histochemistry (UEA and SBA lectins). Alcian Blue $\mathrm{pH} 2.5$ was used as a counterstain; lectins were diluted up to $10 \mu \mathrm{g} / \mathrm{ml}$, and DAB was used as a chromogen. Histochemical grading was conducted blindly according to staining intensity to lectins (0-3). The data was collected and analyzed by the Mann-Whitney U test, using SPSS.

Results: Statistical analysis showed that there was a significant difference between inflammatory mucosa of the bronchial tree and all types of lung cancer (i.e., adenocarcinoma and squamous cell carcinoma, as well as small and large cell lung carcinoma) according to staining intensity to SBA and UEA lectins $(\mathrm{P}<0.001)$. Our results showed that there were many different patterns of reaction to SBA and UEA lectins between all types of lung cancer cells and epithelial cells of the bronchial tree.

Conclusions: Staining intensity and pattern of reaction to lectins were different between all types of lung cancer cells and epithelial mucosa.
\end{abstract}

Keywords: Lung Cancer, Histochemistry, Lectin

\section{Background}

Lung cancer, also known as lung carcinoma, is the most frequent cancer worldwide, and the survival rate of five years is extremely low (15\%) among the people who have this cancer (1). The incidence rate of lung cancer is 1.2 million people per year (2). Lung carcinoma is the most common cause of cancer-related death, accounting for $25 \%$ of overall cancer deaths. Smoking is the most crucial lung cancer risk factor (3). In general, this malignancy can be categorized into Small Cell Lung Carcinoma (SCLC), Adenocarcinoma (ADC), Squamous Cell Carcinoma (SCC), and Large Cell Carcinoma (LCLC) (4). Lung carcinomas are a group of cancers arising from any part of the bronchial tree or pulmonary parenchyma. Thus, lung cancer is a heterogeneous entity both in cell types and biological behavior of specific cell types (5).

ADC arises from the glandular cells of the bronchial mucosa and represents the dominant histological subtype among the other lung cancer types. SCLC arises from the modified bronchial epithelial cells. SCLC is characterized by one of the following specific differentiation features: keratinization, keratin pearl formation, or the presence of intercellular bridges. LCLC is a heterogeneous group of undifferentiated malignant neoplasms that lack cytologic and architectural features of small cell carcinoma 
and glandular or squamous differentiation (6).

Lung cancer is considered the fundamental cause of death among men and the second most serious cause of death among women (7). Cancer progression and metastasis are the most important causes of cancer-related deaths. Extracellular Matrix (ECM) components of the stroma of the tumor play a crucial role in tumorogenesis, cancer progression, and metastasis (8). The functional ability of cancer cells to survive, proliferate, and extend to neighboring cells and distant organs during the process of carcinogenesis is a consequence of altered glycosylation in these cells (9). Glycosylation of the cell surface and ECM components are a type of enzymatic reaction in the process of protein synthesis, which can be catalyzed by glycosyltransferase in the lumen of the endoplasmic reticulum in which some sugars were added covalently to specific amino acids. The terminal sugars can determine the biological and pathological behavior of Cell- ECM interactions (10). Abnormal glycosylation of glycoconjugates can interfere with normal cell proliferation and junctional complexes with neighboring cells, detachments, and cell migration. Cancer cells are continuously shed from their surface glycoconjugates components used for cancer detection in many malignancies (11). Aberrant glycosylation mechanism is the overexpression of glycosyltransferase and glycosidase (12). After the altered glycosylation, cancer progressions are involved in the loss of cell-cell adhesion and initiation of the dissemination of cancer cells. These changes, along with the changes in matrix metalloproteinase, can degrade the physical barrier of ECM, resulting in the invasion and metastasis (13). Sialylation is the process of adding sialic acid to glycoprotein, which changes the half-life of many circulating glycoproteins and has multiple roles in regulating cell-cell, cell-extracellular interactions, and protein targeting. Reportedly, different types of cancers (e.g., breast and cervical cancers) have caused alterations in sialic acid sialidase (14). Fucosylation is an enzymatic process performed by fucosyltransferase, which is one of the most crucial steps in glycoprotein synthesis. Abnormal characteristics of tumor cells include the decreased adhesion and abnormal tumor growth, which can be attributed to the changes in fucosylation of glycoprotein in cancer cells. Studies have shown the importance of monitoring fucosylation during the multistep process of cancer initiation, prognosis, metastasis, early detection, and management of cancer patients (15).

\section{Objectives}

The changes in extracellular matrix glycoconjugates are clear in many tumors, such as glioblastoma, breast cancer, and colorectal carcinoma. O-Glycosylation is one of the crucial posttranslational modifications in nuclear, cytoplasmic, and mitochondrial proteins, which can increase in metastatic cells, thereby highlighting its potential role as a biomarker in cancer (16). Therefore, the current study aimed to detect GlcNac and Fucose containing glycoconjugates in lung cancer.

\section{Methods}

We collected formalin-fixed and paraffin-embedded samples from 25 patients with lung cancer [squamous cell carcinoma $(n=10)$, adenocarcinoma $(n=2)$, inflammatory reaction $(n=10)$ along with large $(n=2)$, and small $(n=1)$ cell lung carcinoma] according to their pathology files at the Ali Ebne-Abitelab Hospital, Zahedan, Iran, during 20152016. Six $\mu$ sections of the samples were obtained and stained with SBA/Alcian Blue pH 2.5, UEA/Alcian Blue pH 2.5, and Hematoxylin-Eosin.

Lectin histochemistry is a microscopy-based technique to visualize cellular components of tissues except that it uses lectin instead of antibodies. The labeled lectins in the tissue staining procedure can limit the technique for the detection of only glycan-conjugated components, as well as those whose glycan moieties are being recognized, particularly by the individual lectins. Unlike immunohistochemistry, which detects the presence of specific antigens based on the specificities of antibodies, lectin histochemistry provides information concerning glycosylation processes within a tissue sample as well as their intracellular locations. This information can be very useful in the characterization and/or the detection of diseases.

According to standard procedures of lectin histochemistry, sections were deparaffinized and hydrated in descending alcohol. HRP conjugated lectins(SBA\&UEA) from Sigma (USA) company were diluted up to $10 \mu \mathrm{gr} / \mathrm{ml}$ in phosphate buffer saline ( $\mathrm{pH}=6.8$ ). Before incubating with lectins, the sections were treated in a $2 \% \mathrm{H}_{2} \mathrm{O}_{2}$ solution in methanol for up to five minutes to neutralize endogenous peroxidase. The sections were then incubated for two hour with the mentioned lectins in the humidified chamber at room temperature. After washing the sections carefully in PBS for $30 \mathrm{~min}$, they were immersed for another $30 \mathrm{~min}$ in a $0.3 \%$ diaminobenzidine (DAB) solution as chromogen containing $0.1 \% \mathrm{H}_{2} \mathrm{O}_{2}$. Afterward, the sections were rinsed again carefully for 30 min under tap water and counterstained using Alcian Blue $\mathrm{pH}=2.5$. They were finally dehydrated, cleared, and mounted based on the routine lab procedure (17). Parallel control sections were used for each patient under the same protocol, except that incubation was not performed with lectins or DAB. Histochemical grading was performed blindly based on staining reactivity in 
three microscopic fields for at least 100 cells as +3 (high reactivity), +2 (moderate reactivity), +1 (low reactivity), and 0 (no reactivity).

The non-parametric Kruskal-Wallis and Mann-Whitney $\mathrm{U}$ tests were used to analyze the data in SPSS software (v. 13). Moreover, histopathological reports were obtained, and photomicrography was performed using the Axiphot Zeiss photomicroscope. The ethical committee of Zahedan University of Medical Sciences approved the study protocol (IR.ZAUMS.REC.1396.328).

\section{Results}

Statistical analysis with Mann Whitney U test using UEA lectin for staining intensity for L-glucose containing glycoconjugates showed a significant difference between inflammatory mucosa with adenocarcinoma, large cell lung carcinoma, and squamous cell carcinoma $(\mathrm{P}<0.001)$. Figure 1 shows the distribution of studied cells according to the degree of reactivity to SBA lectin. Statistical analysis with the Mann Whitney test and with SBA lectin for staining intensity for N-Acetyl glucose amine-containing glycoconjugates demonstrated a significant difference between inflammatory mucosa and Small Cell Lung Carcinoma (P $<0.001$ ). Figure 2 shows the distribution of studied cells according to the degree of reactivity to UEA lectin. Figure 3 demonstrates the pattern and severity of the reaction to UEA, and SBA lectins were different between inflammatory mucosa and all lung cancer types (e.g., large cell lung carcinoma, squamous cell carcinoma, and adenocarcinoma).

\section{Discussion}

Our results showed that the severity of the reaction of cancer cells to lectins (UEA \& SBA) was different between all lung cancer types and inflammatory epithelial mucosal cells. Furthermore, the distribution of cells within different types of lung cancers confirmed the heterogeneous nature of tumoral cells to studied lectins according to the severity of reaction for GlcNac and Fucose containing glycoconjugates. Aberrant glycosylation of glycoproteins and glycolipid is one of the most crucial changes of cancer cells, which is the basis for uncontrolled proliferation and obtains the potential for progression and metastasis in all cancer types (18). Glycosylation is one of the most important biological and pathological actions of all eukaryotic cells that occurs with protein synthesis or posttranslationally in Golgi apparatus on greater than $50 \%$ of proteins, which can alter protein function, biological folding, and physiological functions. It is assumed that altered terminal glycosylation is correlated with tumor progression and metastasis. Chen et al. showed that the expression of $\mathrm{B} 7 \mathrm{H} 3$ was significantly upregulated in tumors of oral squamous cell carcinoma patients and correlated with tumor size, advanced stages, and poor prognosis. B7H3 is a glycoprotein whose N-glycans contain galactose, which is highly fucosylated in oral cancers (19). Cell surface carbohydrates change dramatically during tumorogenesis and affect cancer cell interactions with neighboring cells and extracellular matrix during metastatic spread and tumor growth (20). Analyzing lectin binding in cell surface carbohydrate profiles related to benign and malignant cells can provide a useful tool for the determination of the biological behavior of cancer cells and its potential for growth and metastasis and also become a helpful tool for the determination of patient prognosis (21). Studies showed that critical glycosylation changes of eukaryotic cells occur according to two various processes, namely carbohydrate synthesis blockage or neosynthesis. The analysis of cell surface glycoconjugates in tumor cells demonstrated that the most prevalent alternations in the glycosylation pathway of cancer cells were correlated with the appearance of larger and more branched oligosaccharides (16). Histochemical analysis showed that there were many differences in primary and metastatic lesions in cancer patients. These carbohydrate changes can be a contributing factor to distant metastasis. Studies showed that the inhibition of glycosylation in tumor cells resulted in a profound inhibition of metastasis (22). Carbohydrates of cell surface glycolipid and glycoprotein can change appreciably in all cancer types. Studies showed that specific monoclonal antibodies against colorectal carcinoma, lung carcinoma, and malignant melanoma could detect specific tumor-associated carbohydrates such as CA50. As a ganglioside or sialylated glycoprotein, CA50 has been isolated from primary carcinoma and metastases, and it may be defined as a generalized carcinoma-associated antigen (23). Our results showed that carbohydrate of cell surface glycoconjugates changed in all lung cancer types and secreted carbohydrates of tumor cells at least partly might be from these aberrant glycoconjugates. There are many differences between glycoconjugates content of normal and cancer cells, which may act as a valuable biochemical tool for patient diagnosis and follow-up. Glycosylation analysis showed that there are significant changes in oligosaccharides chains of the secreted product of normal prostate cells and cancer prostate cell lines (LNca P), especially in GalNac, sialic acid, and Fucose content (24). Altered regulation of cell cycle events due to overexpression or amplification of cyclins and cyclin-dependent kinase are the hallmarks of many human-related cancers, such as the esophagus, breast, lung, head and neck, prostate, and colorectal carcinomas (25). Soria et al. demonstrated that cyclin B1 overexpression in lung carcinoma, especially in squamous cell carcinoma, may be a prognostic factor for lung squa- 


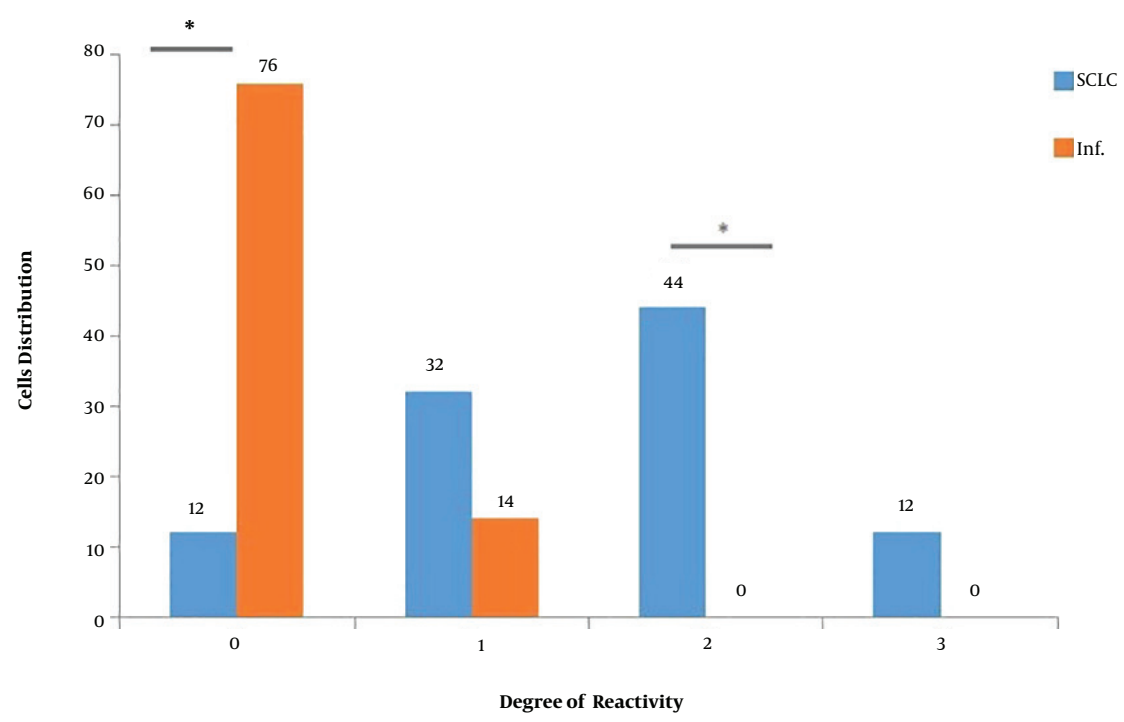

Figure 1. Comparison of the distribution of cells stained to lectin from Soya Bean Agglutinin according to degree of reactivity for GlcNac containing glycoconjugates in inflammatory mucosa (Inf) and Small Cell Lung Carcinoma (SCLC). The horizontal axis shows the histochemical grading according to staining reactivity in three microscopic field: 3 (high reactivity), 2 (moderate reactivity), 1 (low reactivity) and 0 (no reactivity). ${ }^{*} \mathrm{P}<0.001$.

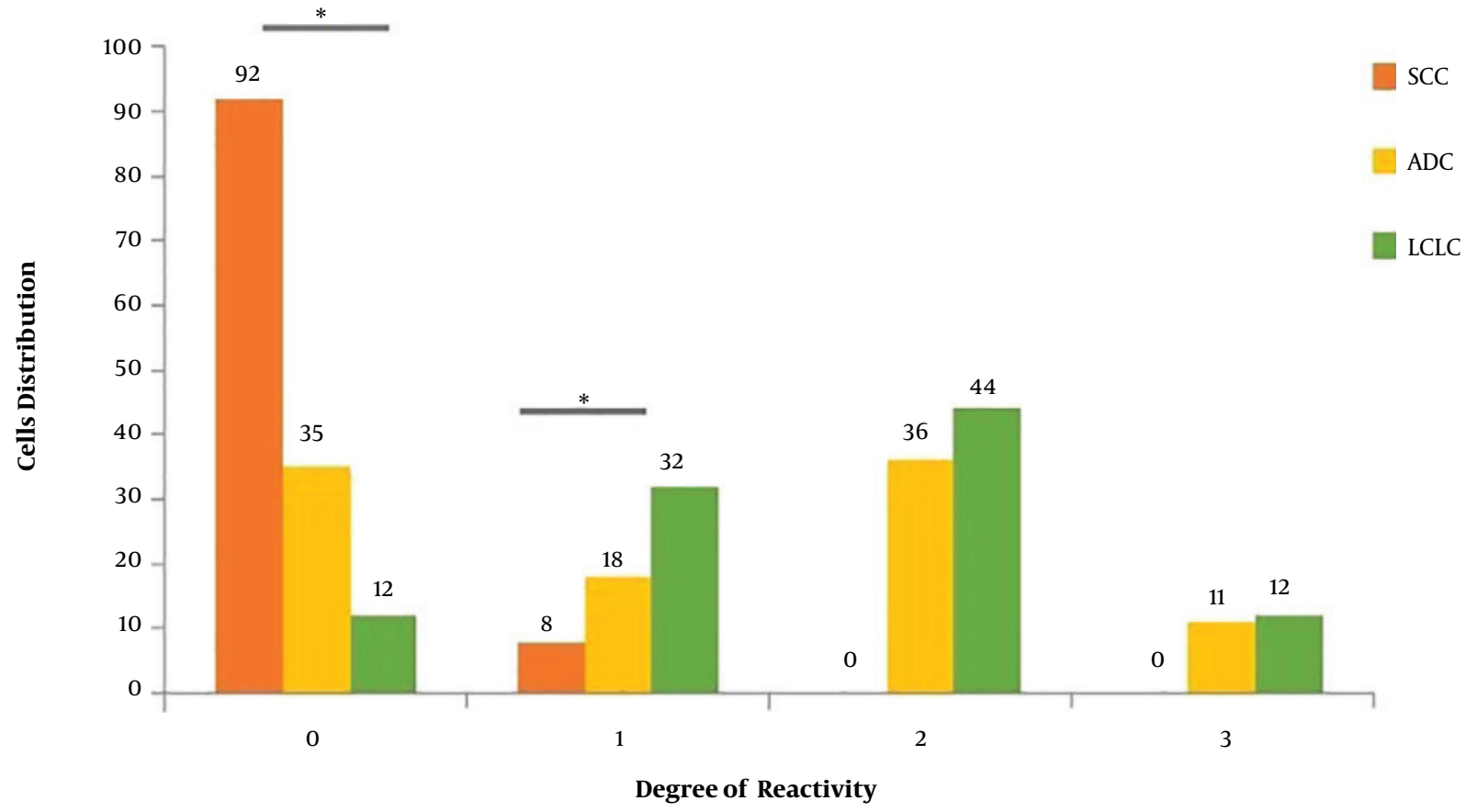

Figure 2. Comparison of the distribution of cells stained to lectin from Ulex Europaeus Agglutinin according to degree of reactivity for Fucose containing glycoconjugates in Squamous Cell Carcinoma (SCC), Adenocarcinoma (ADC) and Large Cell Lung Carcinoma (LCLC). ${ }^{*} \mathrm{P}<0.001$ 

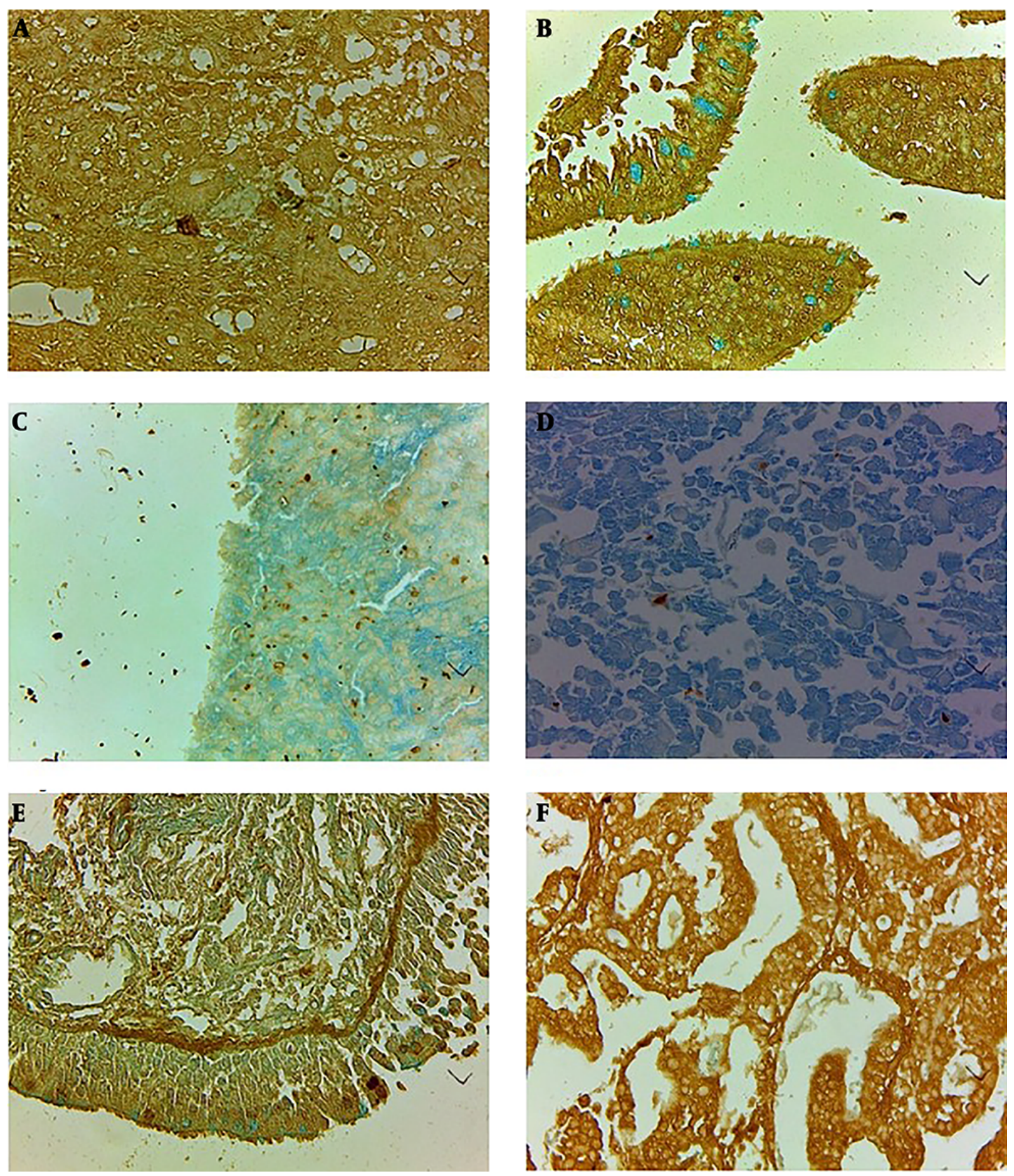

Figure 3. The moderate reaction of tumor stroma and cells of small cell lung carcinoma (a) in comparison to inflammatory mucosa and goblet cells (b) stained to lectin from Soya bean agglutinin (SBA/Alcian Blue), mild reaction of large cell lung carcinoma (c) to lectin from Ulex Europaeus agglutinin and no any reaction of squamous cell carcinoma (d) to lectin was shown. The reaction of inflammatory mucosa to UEA lectin for Fucose containing glycoconjugates restricted to supranuclear regions of columnar cells and also to elastic lamina beneath the epithelium of inflammatory mucosa (e) and moderate to severe staining of adenocarcinoma cells (f) to UEA lectin was clearly shown (magnification: $40 \times$; Scale bar $=250 \mu \mathrm{m}$.). 
mous cell carcinoma. Cytoplasmic reactions of tumor cells to lectins may result from the unscheduled expression of glycoconjugates, which may be a result of either impaired degradation or continued unnecessary synthesis during the cell cycle (26).

\section{Acknowledgments}

The authors of this study are grateful to Zahedan University of Medical Sciences for providing them the required facilities for this research.

\section{Footnotes}

Authors' Contribution: Study concept and design: A.N and M.H.H., Acquisition of data: N.S and S.T and F.S., Drafting of the manuscript and analysis and interpretation of data: Y.A. and M.A and M.A, Critical revision of the manuscript: A.N.

Conflict of Interests: No conflict of interest declared.

Ethical Approval: The ethical committee of Zahedan University of Medical Sciences approved the study protocol (IR.ZAUMS.REC.1396.328).

Funding/Support: None declared.

\section{References}

1. Yang P. Epidemiology of lung cancer prognosis: quantity and quality of life. Methods Mol Biol.2009;471:469-86. doi:10.1007/978-1-59745-4162_24. [PubMed: 19109795]. [PubMed Central: PMC2941142].

2. Lu T, Yang X, Huang Y, Zhao M, Li M, Ma K, et al. Trends in the incidence, treatment, and survival of patients with lung cancer in the last four decades. Cancer Manag Res. 2019;11:943-53. doi: 10.2147/CMAR.S187317. [PubMed: 30718965]. [PubMed Central: PMC6345192].

3. Malhotra J, Malvezzi M, Negri E, La Vecchia C, Boffetta P. Risk factors for lung cancer worldwide. Eur Respir J. 2016;48(3):889-902. doi: 10.1183/13993003.00359-2016. [PubMed: 27174888].

4. Qin J, Lu H. Combined small-cell lung carcinoma. Onco Targets Ther. 2018;11:3505-11. doi: 10.2147/OTT.S159057. [PubMed: 29950855]. [PubMed Central: PMC6016273].

5. Dela Cruz CS, Tanoue LT, Matthay RA. Lung cancer: epidemiology, etiology, and prevention. Clin Chest Med. 2011;32(4):605-44 doi:10.1016/j.ccm.2011.09.001. [PubMed: 22054876]. [PubMed Central: PMC3864624].

6. Zamay TN, Zamay GS, Kolovskaya OS, Zukov RA, Petrova MM, Gargaun A, et al. Current and Prospective Protein Biomarkers of Lung Cancer. Cancers (Basel). 2017;9(11). doi: 10.3390/cancers9110155. [PubMed: 29137182]. [PubMed Central: PMC5704173].

7. North CM, Christiani DC. Women and lung cancer: what is new? Semin Thorac Cardiovasc Surg. 2013;25(2):87-94. doi: 10.1053/j.semtcvs.2013.05.002. [PubMed: 24216523]. [PubMed Central: PMC3827695].

8. Reticker-Flynn NE, Bhatia SN. Aberrant glycosylation promotes lung cancer metastasis through adhesion to galectins in the metastatic niche. Cancer Discov. 2015;5(2):168-81. doi: 10.1158/2159-8290.CD-130760. [PubMed: 25421439]. [PubMed Central: PMC4367955].

9. Vajaria BN, Patel PS. Glycosylation: a hallmark of cancer? Glycoconj J. 2017;34(2):147-56. doi: 10.1007/s10719-016-9755-2. [PubMed: 27975160].
10. Costa J. Glycoconjugates from extracellular vesicles: Structures, functions and emerging potential as cancer biomarkers. Biochim Biophys Acta Rev Cancer. 2017;1868(1):157-66. doi: 10.1016/j.bbcan.2017.03.007. [PubMed: 28347750].

11. Freire-de-Lima L, Previato JO, Mendonca-Previato L. Editorial: Glycosylation Changes in Cancer: An Innovative Frontier at the Interface of Cancer and Glycobiology. Front Oncol. 2016;6:254. doi 10.3389/fonc.2016.00254. [PubMed: 27965934]. [PubMed Central: PMC5124730].

12. Meany DL, Chan DW. Aberrant glycosylation associated with enzymes as cancer biomarkers. Clin Proteomics. 2011;8(1):7. doi: 10.1186/15590275-8-7. [PubMed: 21906357]. [PubMed Central: PMC3170274].

13. Walker C, Mojares E, Del Rio Hernandez A. Role of Extracellular Matrix in Development and Cancer Progression. Int J Mol Sci. 2018;19(10) doi: 10.3390/ijms19103028. [PubMed: 30287763]. [PubMed Central: PMC6213383].

14. Li F, Ding J. Sialylation is involved in cell fate decision during development, reprogramming and cancer progression. Protein \& cell. 2019:116.

15. Jia L, Zhang J, Ma T, Guo Y, Yu Y, Cui J. The Function of Fucosylation in Progression of Lung Cancer. Front Oncol. 2018;8:565. doi: 10.3389/fonc.2018.00565. [PubMed: 30619732]. [PubMed Central: PMC6296341].

16. Peixoto A, Relvas-Santos M, Azevedo R, Santos LL, Ferreira JA. Protein Glycosylation and Tumor Microenvironment Alterations Driving Cancer Hallmarks. Front Oncol. 2019;9:380. doi: 10.3389/fonc.2019.00380. [PubMed: 31157165]. [PubMed Central: PMC6530332].

17. Schumacher DU, Randall CJ, Ramsay AD, Schumacher U. Is the binding of the lectin Helix pomatia agglutinin (HPA) of prognostic relevance in tumours of the upper aerodigestive tract? European Journal of Surgical Oncology (EJSO). 1996;22(6):618-20. doi: 10.1016/s07487983(96)92424-5.

18. Munkley J, Scott E. Targeting Aberrant Sialylation to Treat Cancer. Medicines (Basel). 2019;6(4). doi: 10.3390/medicines6040102. [PubMed: 31614918]. [PubMed Central: PMC6963943].

19. Chen JT, Chen $\mathrm{CH}$, Ku KL, Hsiao M, Chiang CP, Hsu TL, et al. Glycoprotein $\mathrm{B} 7-\mathrm{H} 3$ overexpression and aberrant glycosylation in oral cancer and immune response. Proc Natl Acad Sci U S A. 2015;112(42):13057-62. doi: 10.1073/pnas.1516991112. [PubMed: 26438868]. [PubMed Central: PMC4620862]

20. Schosserer M, Grillari J, Breitenbach M. The Dual Role of Cellular Senescence in Developing Tumors and Their Response to Cancer Therapy. Front Oncol. 2017;7:278. doi: 10.3389/fonc.2017.00278. [PubMed: 29218300]. [PubMed Central: PMC5703792]

21. Nakahara S, Raz A. Biological modulation by lectins and their lig ands in tumor progression and metastasis. Anticancer Agents Med Chem. 2008;8(1):22-36. doi: 10.2174/187152008783330833. [PubMed: 18220503]. [PubMed Central: PMC3794466].

22. Gorelik E, Galili U, Raz A. On the role of cell surface carbohydrates and their binding proteins (lectins) in tumor metastasis. Cancer Metastasis Rev. 2001;20(3-4):245-77. doi: 10.1023/a:1015535427597. [PubMed: 12085965].

23. Holmgren J, Lindholm L, Persson B, Lagergard T, Nilsson O, Svennerholm L, et al. Detection by monoclonal antibody of carbohydrate antigen CA 50 in serum of patients with carcinoma. Br Med J (Clin Res Ed).1984;288(6429):1479-82. doi: 10.1136/bmj.288.6429.1479. [PubMed: 6426610]. [PubMed Central: PMC1441138].

24. Peracaula R, Tabares G, Royle L, Harvey DJ, Dwek RA, Rudd PM, et al. Altered glycosylation pattern allows the distinction between prostatespecific antigen (PSA) from normal and tumor origins. Glycobiology. 2003;13(6):457-70. doi: 10.1093/glycob/cwg041. [PubMed: 12626390].

25. Otto T, Sicinski P. Cell cycle proteins as promising targets in can cer therapy. Nat Rev Cancer. 2017;17(2):93-115. doi: 10.1038/nrc.2016.138. [PubMed: 28127048]. [PubMed Central: PMC5345933].

26. Soria J, Jang SJ, Khuri FR, Hassan K, Liu D, Hong WK, et al. Overexpression of cyclin B1 in early-stage non-small cell lung cancer and its clinical implication. Cancer research. 2000;60(15):4000-4. 